\title{
Piezoelectric Coefficients of PMN-0.33PT Single Crystals
}

\author{
K.C. Cheng', H.L.W. Chan ${ }^{1}$, C.L. Choy ${ }^{1}$, Q.R. Yin ${ }^{2}$, H.S. Luo ${ }^{2}$ and Z.W. Yin ${ }^{2}$. \\ ${ }^{1}$ Department of Applied Physics and Materials Research Centre, The Hong Kong Polytechnic University, \\ Hunghom, Kowloon, Hong Kong, China. \\ ${ }^{2}$ Shanghai Institute of Ceramics, Chinese Academy of Sciences, Shanghai, China.
}

Abstract - Lead magnesium niobate doped with 33 mol\% lead titanate (PMN-0.33PT) single crystals have high piezoelectric coefficients $\left(d_{33}>2000 \mathrm{pC} / \mathrm{N}\right)$ and thus have good potential to be used as sensors, actuators and transducers for medical ultrasound. PMN-0.33PT crystals of diameter $30 \mathrm{~mm}$ and length $40 \mathrm{~mm}$ long have been grown. A sample plate with its normal along the [001] axis was cut from the PMN-PT crystal and was poled along the thickness direction. A sinusoidal voltage was applied across the surfaces of the plate and an interferometer was used to measure the induced displacement in the thickness direction to give the piezoelectric coefficient $d_{33}$. The hydrostatic piezoelectric coefficient $d_{h}$ was also measured and the $d_{31}$ coefficient was calculated by $d_{31}=\left(d_{h}-d_{33}\right) / 2$.

\section{INTRODUCTION}

The superior piezoelectric properties of lead magnesium niobate-lead titanate (PMN-PT) ferroelectric single crystals have attracted considerable research interest in recent years [1-5]. At a PT content of 33 mol\%, PMN-PT crystals have ultrahigh piezoelectric coefficient $\left(d_{33}>2000 \mathrm{pC} / \mathrm{N}\right)$ and electromechanical coupling coefficient $\left(k_{33}>0.9\right)$. For comparison, the corresponding coefficients for the commonly used piezoceramic lead zirconate titanate (PZT) are $d_{33} \sim 300$ $500 \mathrm{pC} / \mathrm{N}$ and $\mathrm{k}_{33} \sim 0.6$. It is thus believed that PMN-x PT $(x=33 \%)$ (abbreviated as PMN-PT in subsequent text) crystals will become a new generation material for transducer, actuator and mechatronic device applications. However, to date, only incomplete data for PMN-PT crystals have been reported [1-5] and the development of practical devices has just begun. In the present study, the piezoelectric coefficients $d_{33}, d_{31}$ and $d_{k}$ of a PMN-PT single crystal grown by the modified Bridgement method [4] have been measured.

\section{EXPERIMENTAL}

A $682 \mu \mathrm{m}$ thick plate with its normal parallel to the [001] axis was cut from a PMN-PT crystal and then poled along the thickness direction. It has an irregular shape and a surface area of $249 \mathrm{~mm}^{2}$. Both sides of the plates are covered with gold electrodes that have mirror-like reflectivity. To determine the $d_{33}$ coefficient, a MachZehnder type heterodyne interferometer (SH-120 from B.M. Industries, France), shown in Fig. 1, was used to measure the displacements in the test sample induced by an a.c. field. A polarized laser beam $L$ with frequency $f_{L}$ and wavelength $\lambda_{L}$ of $632.8 \mathrm{~nm}$ is emitted by a $4 \mathrm{~mW}$ helium-neon (He-Ne) laser source. The complex amplitude of $\mathrm{L}$ is:

$$
L=e^{i 2 \pi \cdot f_{L} \cdot t}
$$

After passing through two deflecting mirrors, the laser beam is split into two half-power beams by a cube beam splitter. A reference beam $R$ is directed through a Dove prism into a photodiode. The frequency of probe beam $P$ is shifted by $f_{B}(70 \mathrm{MHz})$ in a Bragg cell. After reflection from the sample, the phase $\phi(t)$ of probe beam $P$ is modulated by the displacement $d(t)$ of the vibrating surface of the sample. The complex amplitudes of the reference beam $R$ and the probe beam $P$ are:

$$
\begin{aligned}
& R=e^{i 2 \pi \cdot f_{L} \cdot t} \\
& P=p \cdot e^{i 2 \pi \cdot f_{L} \cdot t+i 2 \pi \cdot f_{B} \cdot t+i \phi(t)} \\
& \phi(t)=\frac{4 \pi}{\lambda} d(t)
\end{aligned}
$$

After double passing a quarter-wave plate, the polarization of the reflected beam $S$ is rotated by $90^{\circ}$. The reflected beam $S$ and reference beam $R$ are mixed and then passed through an analyzer at which the polarization of the two orthogonal S \& $R$ beams are oriented by $45^{\circ}$, resulting in the occurrence of interference. The interference of two beams in the photodetector induces photocurrent whose intensity can be expressed as:

$$
I(t)=k \cdot \cos \left(2 \pi f_{B} t+\phi(t)\right)
$$


For a pure sinusoidal displacement of the sample with a single frequency $\omega_{0}$.

$$
\begin{gathered}
d(t)=d_{0} \sin \omega_{0} t \\
I(t)=k \cdot \cos \left(\omega_{B} t+\frac{4 \pi}{\lambda} d_{0} \sin \omega_{0} t\right)
\end{gathered}
$$

which can be expanded into a series of Bessel functions:

$$
\begin{aligned}
& I(t)=\operatorname{Re}\left[e ^ { i \omega _ { B } t } \cdot \left\{J_{0}\left(\frac{4 \pi d}{\lambda}\right)+2 i J_{1}\left(\frac{4 \pi d}{\lambda}\right) \sin \omega_{0} t\right.\right. \\
& \left.\left.+2 J_{2}\left(\frac{4 \pi d}{\lambda}\right) \sin 2 \omega_{0} t+\ldots\right\}\right]
\end{aligned}
$$

The frequency spectrum of the photocurrent induced in a photodetector contains main components at frequency $f_{B}$ and sidebands at $f_{B} \pm f$, where $f$ is frequency of the sample vibration. The absolute vibration displacement can be determined by comparing the components at frequency $f_{B}$ and $f_{B} \pm f$ which can be seen in the spectrum analyzer.

If the displacement $d$ is small compared to $\lambda, d$ is given by:

$$
\frac{J_{1}\left(\frac{4 \pi d}{\lambda}\right)}{J_{0}\left(\frac{4 \pi d}{\lambda}\right)} \cong \frac{2 \pi d}{\lambda}
$$

Applied voltage at $2 \mathrm{kHz}$ was measured by a digital oscilloscope with a $50 \Omega$ termination. The measured displacement as a function of applied voltage is shown in Fig. 2 and from the slope, $d_{33}=$ strain/field $=$ displacement $/$ voltage $=2523 \mathrm{pmV}^{-1}$ is found. The values of $\mathrm{d}_{33}$ measured are independent of frequency until resonance occurs above $50 \mathrm{kHz}$.

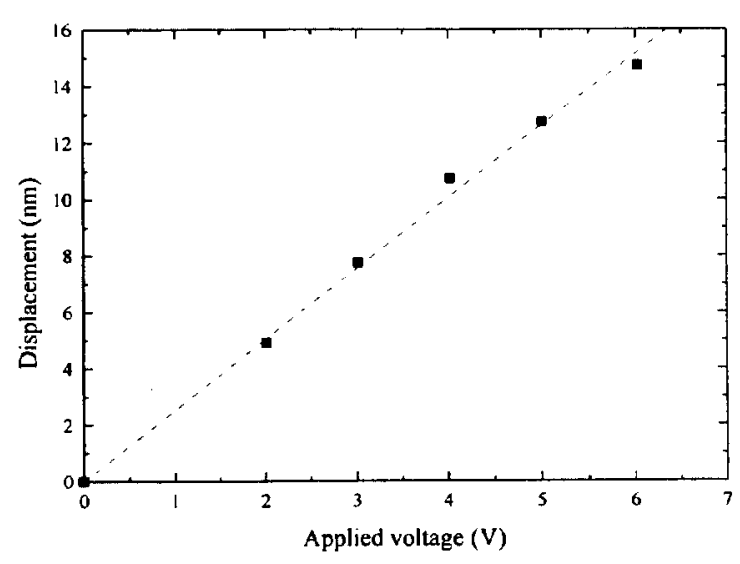

Fig. 2 Displacement against voltage of PMN-PT plate measured at $2 \mathrm{kHz}$.

(slope of the line $=d_{33}$ coefficient)

\section{DETERMINATION OF $d_{h}$ AND $d_{31}$}

The hydrostatic charge coefficients $d_{h}$ is defined as the ratio of the electrical displacement (=charge/area) generated when the sample is subjected to a hydrostatic pressure. $d_{h}$ was measured in a hydrostatic chamber shown in Fig. 3, based on a design by Tancrell et al [6]. The chamber was made of a polymer polymethylmethacrylate (PMMA). Acoustic pressure was generated by two loadspeakers mounted on opposite sides of the chamber. A copper gauze was used to shield the sample located in the middle of the two loadspeakers in order to eliminate the electromagnetic coupling to the signals from the speakers. The loadspeakers were driven by a function generator at a frequency of $80 \mathrm{~Hz}$. At this frequency, the wavelength of the acoustic wave in air is about $4.3 \mathrm{~m}$ so the sample was assumed to be under hydrostatic pressure in the closed cavity.

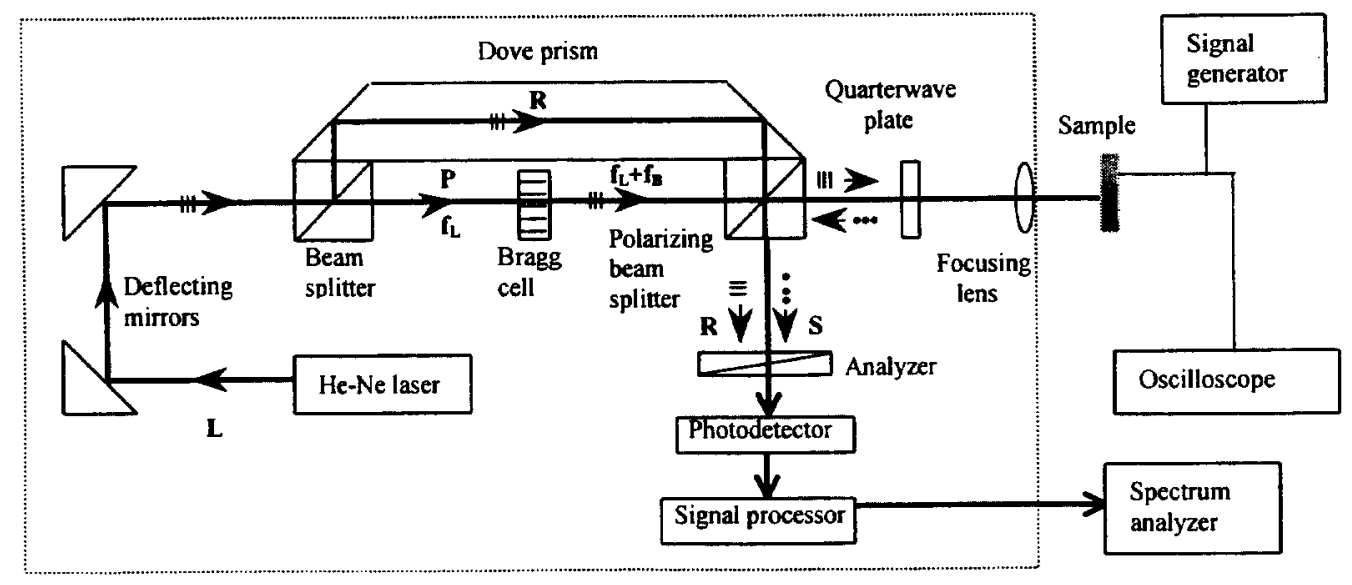

Fig. 1 Experimental setup of the optical measurement. 
The value of $d_{h}$ was determined by measuring the pressure applied to the sample and the charge generated on the two electroded surfaces. The pressure inside the chamber was measured by a calibrated microphone (Bruel \& Kjaer, type 4144). For the calibration of $d_{h}$ measurement, the acoustic pressure was measured as a function of the driving voltage and the result is shown in Fig. 4.

The hydrostatic charge coefficients $d_{h}$ can be calculated by:

$$
d_{h}=\frac{C \cdot V}{P \cdot A}
$$

where $V$ is output voltage from the sample measured by the lock-in amplifier (Standford Research Systems, SR510), $A$ is the electroded area of the sample, $P$ is the acoustic pressure which can be obtained from the calibration curve when the driving voltage is known and $\mathrm{C}$ is the total capacitance at $80 \mathrm{~Hz}$ which is given by:

$$
C=C_{A}+C_{W}+C_{S}
$$

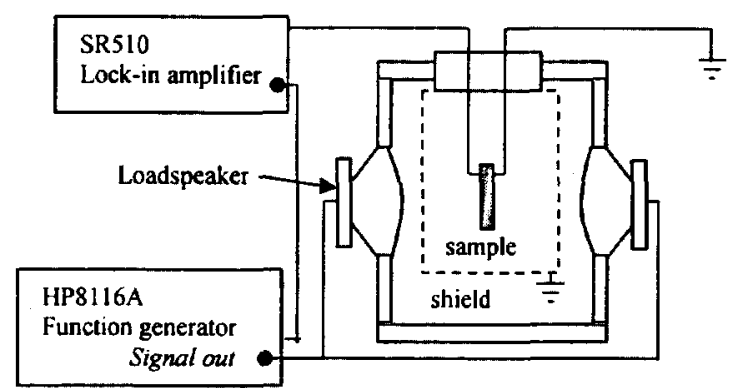

Fig. 3 Experimental setup for measuring the hydrostatic charge coefficient $d_{h}$.

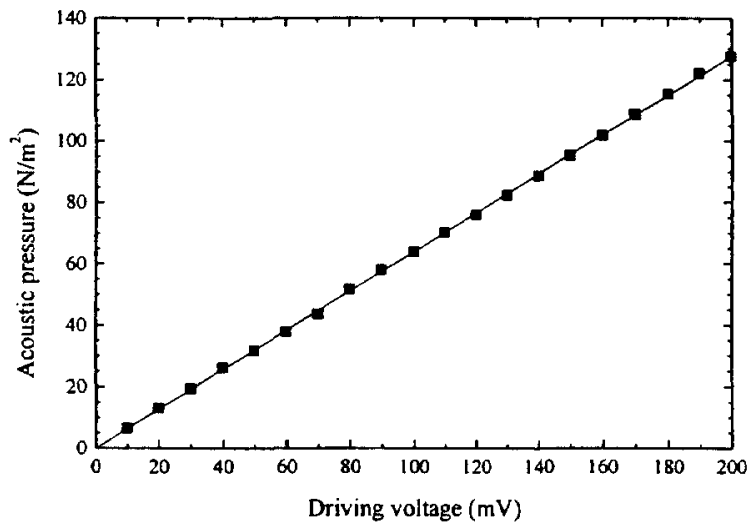

Fig. 4 Calibration curve of the hydrostatic chamber. where $C_{A}$ is the capacitance of the lock-in amplifier $(=25$ $\mathrm{pF}) . \mathrm{C}_{\mathrm{W}}$ is the capacitance of the cable $(=50 \mathrm{pF})$ and $\mathrm{C}_{S}$ is the capacitance of the sample $(=28.74 \mathrm{nF})$. From the $\mathrm{C}_{S}$ value of PMN-PT at $80 \mathrm{~Hz}$, the relative permittivity is found to be 8911. The dielectric loss of PMN-PT is $\tan \delta_{\mathrm{e}}$ $=0.005$, which is rather low. $d_{h}$ is found to be $61 \mathrm{pm} / \mathrm{V}$.

The piezoelectric coefficient $d_{31}$ is calculated from:

$$
d_{31}=\frac{\left(d_{h}-d_{33}\right)}{2}
$$

The $d_{31}$ of PMN-PT is found to be $-1231 \mathrm{pm} / \mathrm{V}$.

\section{OTHER MATERIALS PARAMETERS OF THE PMN-PT SINGLE CRYSTAL}

The electrical impedance and phase of the PMN-PT single crystal as a function of frequency was measured by an impedance analyzer HP 4194A and the thickness mode resonance is shown in Fig. 5. From the equivalent circuit analysis, the mechanical quality factor $\mathrm{Q}_{\mathrm{m}}$ is found to be 69. Following the IEEE Standard for Piezoelectricity [7], the thickness mode electromechanical coupling coefficient $k_{t}$ is found to be 0.61 . Using the antiresonance frequency $f_{n}=3358.25 \mathrm{kHz}$ and a thickness of $682 \mu \mathrm{m}$, the frequency constant $N_{3 t}$ is found to be 2290 $\mathrm{Hz}-\mathrm{m}$.

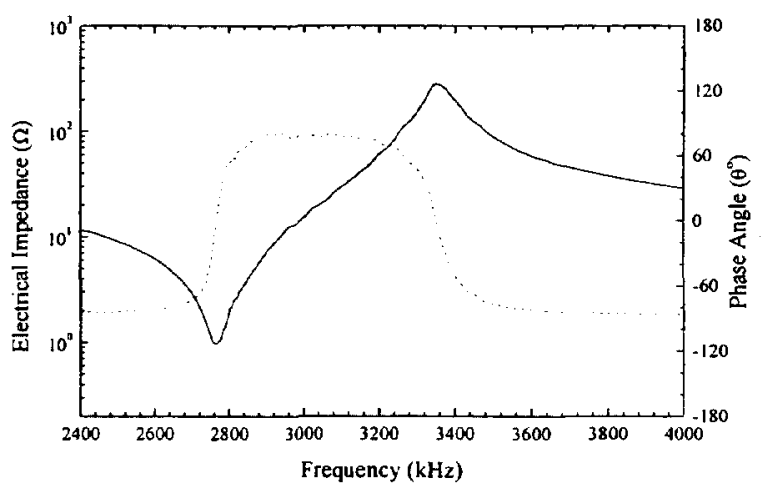

Fig. 5 Thickness mode resonance curve of the PMN-PT plate.

Table I gives a comparison of the measured parameters of the PMN-PT single crystal with PZT8 (hard PZT) and PZT5H (soft PZT) materials [8].

From Table I, it can be seen that the piezoelectric $d_{33}$ and $d_{31}$ coefficients of PMN-PT are much higher than those of PZT. It has a $Q_{m}$ value comparable to that of PZT $5 \mathrm{H}$ but has a higher $\mathrm{k}_{\mathrm{t}}$, thus indicating its advantage in broadband transducer applications. 
Table I Material parameters of PMN-PT, PZT 8 and PZT 5H.

\begin{tabular}{|l|l|l|l|l|l|l|l|l|}
\hline & $d_{33}(\mathrm{pm} / \mathrm{V})$ & $\mathrm{d}_{31}(\mathrm{pm} / \mathrm{V})$ & $\mathrm{d}_{\mathrm{h}}(\mathrm{pm} / \mathrm{V})$ & $\varepsilon_{33}{ }^{\mathrm{T}}$ & $\tan \delta_{\mathrm{e}}$ & $\mathrm{k}_{\mathrm{t}}$ & $\mathrm{Q}_{\mathrm{m}}$ & $\begin{array}{l}\mathrm{N}_{3 \mathrm{t}} \\
(\mathrm{Hz.m})\end{array}$ \\
\hline PMN-PT & 2523 & -1231 & 61 & 8911 & 0.005 & 0.61 & 69 & 2290 \\
\hline PZT8 & 225 & -97 & 31 & 1000 & 0.004 & 0.48 & 1000 & 2070 \\
\hline PZT 5H & 593 & -274 & 45 & 3400 & 0.025 & 0.50 & 65 & 2000 \\
\hline
\end{tabular}

\section{CONCLUSION AND DISCUSSION}

The PMN-PT sample studied in the present work was cut from one of the top-quality crystals grown at the Shanghai Institute of Ceramics, China. However, additional work is required to grow crystals with good homogeneity and consistently high piezoelectric coefficients. As the PMN-PT crystal has high $d_{33}$ and $d_{31}$ coefficients, it is imperative to find applications that can fully exploit both of these high values. The cymbal actuator [9] is one possible application as it couples the $d_{33}$ and $d_{31}$ to give a high displacement. We have done some preliminary work which shows that PMN-PT cymbals can give displacements several times higher than those of PZT cymbals and the results will be reported in the near future.

\section{ACKNOWLEDGEMENT}

The work described in this paper was supported by the Research Grants Council of the Hong Kong Special Adminstrative Region (Project No. PolyU 5193/00P), the Industrial Support Fund ISF (Project No. AF/147/98) and the Centre for Smart Materials of the Hong Kong Polytechnic University.

\section{REFERENCES}

[1] O. Noblanc, P. Gaucher and G. Calvarin, "Structural and dielectric studies of $\mathrm{Pb}\left(\mathrm{Mg}_{\mathrm{I} / 3} \mathrm{Nb}_{2 / 3}\right) \mathrm{O}_{3}-\mathrm{PbTiO}_{3}$ ferroelectric solid solutions around the morphotropic boundary", $J$. Appl. Phys., Vol.79(8), pp.4291-4297 (1996).

[2] H. Ouchi, J. Am. Ceram. Soc., Vol.51, pp.169 (1968).

[3] T.R. Shrout, Z.P. Chang, N. Kim , S. Markgraf, "Dielectric behavior of single crystals near the (1x) $\mathrm{Pb}\left(\mathrm{Mg}_{1 / 3} \mathrm{Nb}_{2 / 3}\right) \mathrm{O}_{3}$-(morphotropic phaseboundary", Ferroelectrics Letts, Vol.12, pp.63-69 (1990).

[4] H. S. Luo, G. S. Xu, P. C. Wang,, Z. W. Yin, "Growth and characterization of relaxor ferroelectric PMNT single crystals", Ferroelectrics, Vol.231, pp.685-690 (1999).

[5] W. G. Luo, A. Ding, H. Luo, G. Xu and Z. Yin, "High-field properties of PMN-PT single crystals", Proc. 1999 IEEE Ultraonics Symp., Nevada, USA, Oct., Vol.2, pp.1009-1012 (1999).

[6] R. H. Tancrell, D. T. Wilson, D. Rickette, "Properties of PVDF polymer for sensor," Ultrasonic Symposium, pp.624-629 (1985).

[7] IEEE Standard on Piezoelectricity, ANSI/IEEE Std. 176-1987.

[8] Piezoelectric ceramics data book for designers, Morgan Matroc Limited, England.

[9] J. F. Fernandez, A. Dogan, J. T. Fielding, K. Uchino, R. E. Newnham, "Tailoring the performance of ceramic-metal piezocomposite actuators, Cymbals", Sensors \& Actuators, A65, pp. 228-237 (1998). 